\title{
Immediate effect of vagotomy on pancreatic insulin secretion
}

I Nordback, E Harju

\begin{abstract}
The effect of vagotomy and gastric resection on insulin secretion was examined by the glucagon stimulated C-peptide test in gastrectomy patients $(n=11)$ without truncal vagotomy and in total gastrectomy patients $(n=10)$ with truncal vagotomy. The test was performed twice in each patient: 10 minutes after the midline incision was made and then $\mathbf{6 0}$ to 90 minutes later when gastric resection or total gastrectomy was completed, during the reconstructive phase of the operation. Gastric resection without truncal vagotomy was followed by a higher increase $(\mathbf{4 8 \%})$ in serum Cpeptide concentration caused by glucagon stimulation than total gastrectomy with truncal vagotomy $(13 \%)$. There was a significant $(p<0.05)$ increase in the glucagon stimulated glucose-related $\mathrm{C}$-peptide concentration in patients without truncal vagotomy, whereas truncal vagotomy inhibited this increase. These results suggest that truncal vagotomy will produce a reduction in stimulated insulin secretion in humans.
\end{abstract}

Total gastrectomy is frequently followed by complaints which are thought to be caused, at least in part, by abnormal glucose absorption and metabolism. ${ }^{12}$ Vagotomy may also affect glucose metabolism as a less rapid rise and a lower peak concentration of blood glucose have been found before vagotomy than after vagotomy in patients without gastric resection. ${ }^{3}$ This has been explained firstly by an increase in intestinal glucose absorption occurring after an adaptation period of five to six weeks, ${ }^{4}$ and secondly by a decreased response of insulin to glucose load, especially to oral glucose. ${ }^{3}$ Malabsorption of glucose $^{5}$ and altered response of indirect mediators such as intestinal hormones have also been offered as explanations. ${ }^{3}$ There are also findings that suggest a direct effect of the vagus nerve on the endocrine pancreas ${ }^{3}$; similar peripheral venous plasma insulin concentrations have been found after intravenous glucose infusions in patients with truncal, selective, and highly selective vagotomy, but blood glucose concentrations were significantly higher in patients with truncal vagotomy. ${ }^{3}$ Because of the rapid metabolism of insulin in the liver the peripheral insulin concentration may not fully explain the response of pancreatic insulin secretion to glucose. To study this, the glucagon stimulated C-peptide test was used to compare pancreatic insulin secretion capacity before and after truncal vagotomy.

Patients and methods

Because truncal vagotomies alone are not per- formed in our hospital due to postoperative complications,${ }^{6}$ patients with a total gastrectomy plus truncal vagotomy were chosen for study (10 patients). The control group consisted of patients with resection of the stomach without truncal vagotomy (11 patients) (Table). The reconstruction was a Roux-Y-loop in all the cases. None of the patients had a known defect in, or had had treatment that might have interfered with glucose metabolism. Signed informed consent was obtained from all patients. The study was accepted by the Ethical Committee of Tampere University Central Hospital.

A standardised general combined anaesthesia was used. The following tests were carried out in every patient during the operation. Ten minutes after the incision into the abdomen the first glucagon stimulated C-peptide test (test A) was performed. Sixty to 90 minutes later, when the resection had been completed (during the reconstructive phase of the operation), the second glucagon stimulated C-peptide test was performed (test B).

In both tests the first blood sample was taken from a peripheral vein in the arm opposite to the one used for intravenous infusion, to determine the base concentrations of blood glucose and serum C-peptide, ${ }^{7}$ and $1 \mathrm{mg}$ glucagon was given intravenously immediately. Six minutes later the second blood sample was taken to determine concentrations of glucose and C-peptide after stimulation, the latter reflecting the beta cell function. ${ }^{89}$

\section{Results}

There was an increase in both serum C-peptide and blood glucose concentration after glucagon stimulation before and after gastric resection with or without vagotomy (Fig 1 A and B). There were no significant differences between the two study groups. In the gastrectomy with vagotomy group, however, glucagon stimulation caused only a $13 \%$ higher increase in serum C-peptide concentration in test $B$ than in test $A$, while the increase was $48 \%$ in the non-vagotomy group.

There was a significant $(p<0.05)$ increase in the glucagon stimulated glucose-related C-pep-

Details of patients in both study groups (I total gastrectomy and truncal vagotomy; II resection of the stomach without truncal vagotomy

\begin{tabular}{lll}
\hline & $I$ & $I I$ \\
\hline No of patients & 10 & 11 \\
Men/women & $4 / 6$ & $7 / 4$ \\
Age (years; mean, range) & $62(41-79)$ & $66(48-80)$ \\
Operated on for: & & \\
$\quad$ Gastric cancer & 10 & 4 \\
Gastric ulcer & - & 7 \\
Overweight $>15 \%$ & 3 & 5 \\
\hline
\end{tabular}

Sciences, University of

Tampere, Finland

E Harju

Correspondence to:

Dr Isto Nordback

Institute of Clinical Sciences,

Tampere University, 33520

Tampere, Finland.

Accepted for publication

23 April 1990 

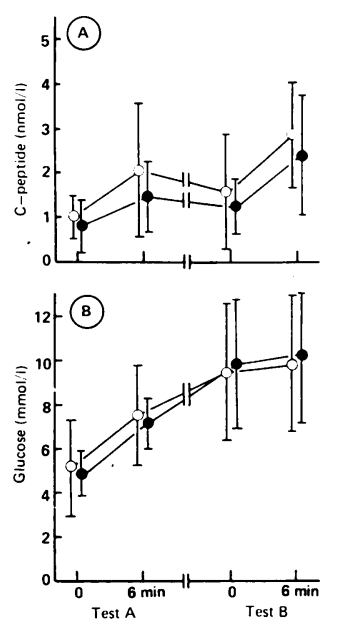

Figure 1: (A) Serum $C$-peptide and $(B)$ blood glucose concentrations in patients with gastric resection with (O) or without (O) truncal vagotomy before and 6 minutes after glucagon stimulation both before (test $A)$ and after (test B) gastric resection. Mean $(S D)$. tide concentration in patients without truncal vagotomy, whereas truncal vagotomy inhibited this increase (Fig 2).

\section{Discussion}

Most of the work on the vagal effects on pancreatic endocrine function has been experimental studies in animals. ${ }^{10}$ These have shown that truncal vagotomy abolishes circadian rhythms in basal glucose and insulin levels" and causes abnormalities in oral glucose tolerance tests. ${ }^{112}$ Unlike unoperated rats or rats with selective gastric vagotomy, rats with truncal vagotomy experienced glucosuria after glucose ingestion. ${ }^{13}$ Moreover, intravenous glucose load has led to lower plasma insulin concentrations in peripheral blood after pyloroplasty with truncal vagotomy than after pyloroplasty alone. ${ }^{12}$ Thus truncal vagotomy alone or in combination with gastrectomy might explain in part the postgastrectomy complaints associated with altered glucose metabolism.

In human studies oral glucose intake, but not intravenous glucose load, has been shown to cause a lower response in the peripheral venous plasma insulin concentration in patients with truncal vagotomy than in those with a selective or highly selective vagotomy. ${ }^{3}$ The lower response of plasma insulin to oral glucose after truncal vagotomy becomes obvious only after 90 minutes after glucose ingestion, ${ }^{14}$ suggesting that some indirect mediators are involved. Moreover, some authors have totally neglected the possible effect of the parasympathetic nervous system on insulin secretion. ${ }^{15}$

In the earlier work on the effect of vagotomy on glucose metabolism plasma insulin concentrations have served as the indicator for pancreatic insulin secretion. The plasma insulin concentration in peripheral veins is not an optimal parameter in this respect, however, because after secretion from the pancreas into the portal blood insulin is rapidly degraded in the liver before entering the peripheral circulation. In peripheral blood the half life of insulin is short ( 10 minutes). C-peptide is the peptide which is split from proinsulin when active insulin is formed in the pancreas. C-peptide is secreted into portal blood in the same proportion as insulin. Unlike insulin, however, it is metabolised in the kidneys instead of the liver and has a half life of 20 minutes. In peripheral blood the $\mathrm{C}$-peptide concentration is ten times higher than the insulin concentration. ${ }^{9}$ Thus the C-peptide concentration in peripheral blood reflects the pancreatic insulin secretion into the portal blood. This is why we have used serum C-peptide assays instead of insulin in our studies.

Earlier we obtained results which suggest that there is no absolute defect in insulin secretion capacity after total gastrectomy but there may instead be a relative lack of insulin during postprandial hyperglycaemia caused by glucose intake. ${ }^{16}$ Moreover, our experiment in healthy subjects showed that chemical vagotomy by atropine decreased the glucagon stimulated insulin secretion of the pancreas. ${ }^{17}$

In this study there was again no measured differences in intraoperative absolute unstimu- lated or glucagon stimulated C-peptide concentrations between the patients with and those without vagotomy. Nearly a 50\% higher C-peptide response to glucagon was found, however, after gastric resection as tested peroperatively. This was strongly inhibited by truncal vagotomy, for only a $13 \%$ increase was found during gastrectomy with truncal vagotomy. Also blood glucose concentration related C-peptide response to glucagon was significantly higher after gastric resection without vagotomy, whereas no such difference was found in patients with additional truncal vagotomy. These results support the concept of the diminishing effect of vagal denervation on stimulated pancreatic insulin secretion. This might be one explanation for the altered glucose metabolism after gastric surgery and vagotomy.

It has been suggested that intestinal hormones act as mediators of altered glucose metabolism after gastric operations. ${ }^{3}$ On the other hand, pancreatic polypeptide has been shown to alter glucose metabolism by affecting the hepatic response to insulin. ${ }^{18-20}$ The secretion of this hormone is thought to be regulated both directly neurally ${ }^{21}$ and neurally through antral polypeptide releasing factor..$^{22}$ Thus alterations in postgastrectomy glucose metabolism might also be mediated through pancreatic polypeptide. In the present study pancreatic polypeptide was not assayed. Pancreatic polypeptide, however, cannot act alone as a mediator, since diminution in blood glucose concentration related glucagonstimulated insulin secretion capacity can be detected immediately after vagotomy during the operation. Within 20 minutes, however, this altered insulin response may cause changes in pancreatic polypeptide secretion with subsequent pronounced effects of glucose metabolism. ${ }^{23}$

In our hospital truncal vagotomy is performed only in conjunction with total gastrectomy. In the control patients without vagotomy gastric resection entailed resecting about two thirds of the stomach. Thus the present patients were different not only in respect of the vagotomy but also the extent of the gastric resection. Apart from this difference, these results suggest that truncal vagotomy will produce an appreciable change in the insulin response in man.

1 Le Quesne LP, Hobsley M, Hand BH. The dumping syndrome. I. Factors responsible for the symptoms. $\mathrm{Br} \mathrm{Med} \mathcal{f}$ 1960; i: 141-7.

2 Hobsley M, Le Quesne LP. The dumping syndrome. II Cause of the syndrome and the rationale of its treatment $\mathrm{BrMed}$ F 1960; i: 147-51.

3 Humphrey CS, Dykes JR, Johnston D. Effects of truncal vagotomy on glucose tolerance and insulin secretion in patients with duodenal ulcer. Br Med F 1975; ii: 112-6.

4 Andrews NJ. Acute and chronic effects of truncal vagotomy upon glucose absorption, water and electrolyte fluxes in the small intestine of the rabbit. Surg Gynecol Obstet 1985; 161: 33-6.

5 Radziuk J, Bondy DC. Abnormal oral glucose tolerance and glucose malabsorption after vagotomy and pyloroplasty. A glucose malabsorption after vagotomy and pyloroplasty. A tracer method for measuring glus
Gastroenterology 1982; 83: 1017-25.

6 Alexander-Williams J. Complications of gastric surgery. In Bouchier IAD, Allan RN, Hodgson HJF, Keighley MRB eds. Textbook of gastroenterology. London: Baillière Tindall, 1984: 201-17

7 Heding LG. Radioimmunological determination of human C-peptide in serum. Diabetologia 1975; 11: 541-8.

8 Faber OK, Binder C. C-peptide response to glucagon. A test for the residual beta-cell function in diabetes mellitus. Diabetes 1977; 26: 605-10.

9 Faber OK, Hagen C, Binder C. Kinetics of human connecting peptide in normal and diabetic subjects. $\mathcal{F}$ Clin Invest 1973; 62: 197-203. 
10 Campfield LA, Smith FJ, LeMagnen J. Altered endocrine pancreatic function following vagotomy: possible behavioral pancreatic function following vagotomy: possible behavioral and metabolic bases for assessing comp

11 Louis-Sylvestre J. Feeding and metabolic patterns in rats with truncular vagotomy or with transplanted B-cells. Am f Physiol 1978; 235: 119-25.

12 Hakanson R, Liedberg G. Lundquist I. Effect of vagal denervation on insulin release after oral and intravenous glucose. Experientia 1971; 27: 460-1.

13 Louis-Sylvestre J, Giachetti I, Le Magnen J. A non-invasive test for completeness of vagotomy to the pancreas. Physiol Behav 1981; 26: 1125-7.

14 Russel RCG, Thomson JPS, Bloom SR. The effect of truncal and selective vagotomy on the release of pancreatic glucagon, insulin and enteroglucagon. Br $\mathcal{F}$ Surg 1974; 61: 821-4.

15 Linquette M, Fourlinnie JC, Lagache G. Étude de la glycémie et de l'insulinémie après vagotomie et pyloroplastie chez et de l'insulinémie après vagotomie et pyloroplaste
l'homme. Ann Endocrinol (Paris) 1969; 30: 96-102.

16 Harju E, Nordback I. Blood C-peptide concentration during post-prandial hyperglycemia in patients after total gastrectomy. Panminerva Med 1985; 27: 71-4.
17 Harju E, Nordback I. Effect of atropine on insulin secretion in healthy subjects. F Int Med Res 1987; 15: 167-9.

18 Hazelwood RL. Synthesis, storage, secretion, and significance of pancreatic polypeptide in vertebrates. In: Cooperstein SJ, Watkins $\mathrm{D}$, eds. The islet of Langerhans. Biochemistry, physiology, and pathology. New York: Academic Press, 1981 275-318.

19 Floyd JC Jr. Human pancreatic polypeptide. In: Buchanan $\mathrm{KD}$, ed. Clinics in endocrinology and metabolism. Vol 8. Philadelphia: Saunders WB, 1977: 379-94.

20 Bloom SR, Adrian TE, Greenburg GR. Effects of pancreatic polypeptide infusion in man. Diabetes 1977; 26: 375 .

21 Prinz RA, Sabbagh HE, Adrian TE, et al. Neural regulation of pancreatic polypeptide release. Surgery 1983; 94: 1011-8.

22 Debas HT, Taylor IL, Seal AM, Passaro EP. Evidence for vagus-dependent pancreatic polypeptide-releasing factor in the antrum: studies with autotransplanted dog pancreas. the antrum: studies with
Surgery 1982; 92: 309-14.

23 Brunicardi FC, Druck P, Sun YS, Elahi D, Gingerich RL, Andersen DK. Regulation of pancreatic polypeptide secretion in the isolated perfused human pancreas. Am $\mathcal{F}$ Surg 1988; 155: 63-9. 